Pacific Journal of Mathematic 


\title{
ON PRODUCTS OF MAXIMALLY RESOLVABLE SPACES
}

\section{J. Ceder and T. Pearson}

\begin{abstract}
A maximally resolvable space is one which can be decomposed into the largest number of "maximally dense" subsets. Answering a previously posed question, we show that an arbitrary product of maximally resolvable spaces is again maximally resolvable, not only with respect to the ordinary product topology, but with respect to other natural topologies as well.
\end{abstract}

Given a topological space $(X, \tau)$ let $\Delta(X, \tau)$ denote the least among the cardinals of nonvoid $\tau$-open sets. A space $(X, \tau)$ is said to be maximally resolvable if it has isolated points or $X$ is the union of $\Delta(X, \tau)$ pairwise disjoint sets, called resolvants, each of which intersects each nonvoid open set in at least $\Delta(X, \tau)$ points.

In [1] the first author showed among things that locally compact Hausdorff spaces and first countable spaces are always maximally resolvable. Moreover, it was shown that in certain cases the product of maximally resolvable spaces is maximally resolvable. In this paper we settle this question of the maximal-resolvability of products by showing that an arbitrary product of maximally resolvable spaces is maximally resolvable with respect to the ordinary product topology, the generalized product topology, and the box topology. Other results, as well as some interesting unsolved problems regarding products of maximally resolvable spaces, are also presented.

In the sequel we will consider ordinals and cardinals defined so that each ordinal is equal to the set of its predecessors, and a cardinal is an ordinals which is not equipollent with any smaller ordinal. Cardinals will be denoted by the aleph notation or by the symbols $\mathbf{k}, \mathrm{m}, \mathbf{n}$, etc., and ordinals will be denoted by lower case Greek letters $\alpha, \beta, \gamma$, etc.. The cardinal number of a set $A$ will be denoted by $|A|$. A subset $B$ of a topological space $X$ is said to be $m$-dense in $X$ if $|B \cap U| \geqq \boldsymbol{m}$ for each nonvoid open subset $U$ of $X$. A subset $B$ of a given set $M$ is said to be small (resp. large) with respect to $M$ if $|B|<|M|$ (resp. $|M-B|<|M|$ ). When no confusion is likely, we will denote $\Delta(X, \tau)$ by $\Delta(X)$.

Given a Cartesian product $\Pi\left\{X_{\alpha}: \alpha \in M\right\}$ of topological spaces $X_{\alpha}$, let $\mathscr{U}$ be the collection of all sets of the form $\Pi\left\{U_{\alpha}: \alpha \in M\right\}$ where $U_{\alpha}$ is open in $X_{\alpha}$. Let $\mathscr{B}$ be the topology generated by the base $\mathscr{U}$. Let $\mathscr{P}($ resp. $\mathscr{G}$ ) be the topology generated by the base consisting of all members of $\mathscr{U}$ for which $U_{\alpha}=X_{\alpha}$ except for finitely many 
indices (resp. $U_{\alpha}=X_{\alpha}$ except for a small subset of indices [here, we assume $\left.\left.\boldsymbol{\aleph}_{0} \leqq|M|\right]\right)$. Then $\mathscr{B}, \mathscr{P}$ and $\mathscr{G}$ will be called the box, ordinary product, and generalized product topologies respectively.

2. The ordinary product topology. In this section we will consider only product spaces equipped with the ordinary product topology $\mathscr{P}$. We begin with two lemmas.

Lemm 1. (Ceder [1]) A finite product of maximally resolvable spaces is maximally resolvable.

LeMma 2. If $X$ is maximally resolvable with $\Delta(X) \neq 1$ and $Y$ is such that $\Delta(Y) \leqq \Delta(X)$, then $X \times Y$ is maximally resolvable.

Proof. Since $\Delta(X)$ is necessarily infinite, we have $\Delta(X \times Y)=\Delta(X) \cdot \Delta(Y)=\Delta(X)$. If $\left\{X_{\alpha}: \alpha<\Delta(X)\right\}$ is a collection of resolvants for $X$, than obviously $\left\{X_{a} \times Y: a<\Delta(X)\right\}$ gives a maximal resolution for $X \times Y$.

If $X$ and $Y$ are related as in the hypothesis of Lemma 2, then we will say that $X$ dominates $Y$. This terminology will be used repeatedly in the proofs in the sequel. Next we have our basic theorem.

Theorem 1. Suppose $\boldsymbol{m} \geqq \boldsymbol{\aleph}_{\circ}$ and $\left|X_{\alpha}\right| \geq 2$ for each $\alpha<\boldsymbol{m}$. If

$$
\sup _{\alpha \in \boldsymbol{m}}\left|X_{\alpha}\right| \leqq \Delta\left(\Pi\left\{X_{\alpha}: \alpha<\boldsymbol{m}\right\}\right) \leqq\left|\Pi\left\{X_{\alpha}: \alpha \notin A\right\}\right|
$$

for each finite subset $A$ of $\boldsymbol{m}$, then $\Pi\left\{X_{\alpha}: \alpha<\boldsymbol{m}\right\}$ is maximally resolvable.

Proof. Let

$$
\mathscr{A}=\cup\left\{X_{\alpha_{1}} \times X_{\alpha_{2}} \times \cdots \times X_{\alpha_{n}}:\left\{\alpha_{1}, \alpha_{2}, \cdots, a_{n}\right\} \text { a finite subset of } \boldsymbol{m}\right\} \text {. }
$$

Denote $\Delta\left(\Pi\left\{X_{\alpha}: \alpha<\boldsymbol{m}\right\}\right)$ by $\boldsymbol{n}$ and put $W=\mathscr{A} \times \boldsymbol{n} \times \boldsymbol{n}$. Clearly $|\mathscr{A}| \leqq \boldsymbol{n} \cdot \boldsymbol{m}=\boldsymbol{n}$ so that $|W|=\boldsymbol{n}$. Thus we can well-order $W$ as $\left\{w_{\alpha}\right\}_{\alpha<n}$. We shall now define the desired resolvants for the product by means of transfinite induction on the ordinal $\boldsymbol{n}$.

Suppose $f_{\gamma}$ has been chosen for each $\gamma<\beta$ and consider $w_{\beta}=$ $\langle A, \mu, \nu\rangle$ where $A=\left\langle x_{\alpha_{1}}, x_{\alpha_{2}}, \cdots, x_{\alpha_{n}}\right\rangle \in \mathscr{A}$. Put

$$
C_{\boldsymbol{A}}=\left\{f \in \Pi\left\{X_{\alpha}: \alpha<\boldsymbol{m}\right\}: f\left(\alpha_{i}\right)=x_{\alpha_{i}}\right\} .
$$

Since $\boldsymbol{n} \leqq\left|\Pi\left\{X_{\alpha}: \alpha \notin A\right\}\right|$ we have $\left|C_{A}\right| \geqq \boldsymbol{n}$, so that $C_{A}-\left\{f_{\gamma}: \gamma<\beta\right\} \neq$ $\varnothing$. Now pick $f_{\beta} \in C_{A}-\left\{f_{\gamma}: \gamma<\beta\right\}$. 
Now, having defined the set of distinct points $\left\{f_{\alpha}\right\}_{\alpha<n}$, we define for each $\alpha<\boldsymbol{n}$ the set $B_{\alpha}=\left\{f_{\beta}\right.$ : there exists $A \in \mathscr{A}$ and $\gamma<\boldsymbol{n}$ such that $\left.w_{\beta}=\langle A, \alpha, \gamma\rangle\right\}$. Obviously, $\left|B_{\alpha} \cap C_{A}\right|=\boldsymbol{n}$ for each $A \in \mathscr{A}$ and $\alpha<\boldsymbol{n}$, so that each $B_{\alpha}$ is $\boldsymbol{n}$-dense. Hence, $\left\{B_{\alpha}\right\}_{\alpha<\boldsymbol{n}}$ is a family of $\boldsymbol{n}$ pairwise disjoint, $\boldsymbol{n}$-dense subsets of $\Pi\left\{X_{\alpha}: \alpha<\boldsymbol{m}\right\}$ so that $\Pi\left\{X_{\alpha}: \alpha<\boldsymbol{m}\right\}$ is maximally resolvable.

As a corollary of the above result it follows that an infinitefold product of any space with itself is maximally resolvable.

The technique of the proof of Theorem 1 suggests a way of obtaining a sufficient condition for the maximal-resolvability of an infinite completely regular space $X$, namely: embed $X$ in $\beta X$, define $C_{A}$ and $\mathscr{A}$ in the analogous manner, and duplicate the proof of Theorem 1. The condition essential for the success of this method is that $|\mathscr{C}| \leqq A(X)$, where $\mathscr{C}$ is the family of all continuous functions from $X$ into $[0,1]$. But since $\chi(X) \leqq|\mathscr{E}|$ this condition implies $\chi(X) \leqq \Delta(X)$, where $\chi(X)$ is the weight of $X$ (i.e., the least among the cardinals of bases of $X)$. However, Theorem 3 of [1] asserts that any space $X$ is maximally resolvable whenever $\boldsymbol{\aleph}_{0} \leqq \chi(X) \leqq \Delta(X)$. Therefore, the method of Theorem 1 applied to the image of $X$ in $\beta X$ gives no new results.

We remark in passing that the techniques of the proof of Theorem 1 yield a proof of Theorem 3 of [1] which is much simpler than the original.

THeOREM 2. If $X_{\alpha}$ is maximally resolvable for each $\alpha<\boldsymbol{m}$, then $\Pi\left\{X_{\alpha}: \alpha<\boldsymbol{m}\right\}$ is maximally resolvable.

Proof. In view of Lemma 1 we can assume that $\boldsymbol{m}$ is infinite and each $\left|X_{\alpha}\right| \geqq 2$. Let $\boldsymbol{n}=\Delta\left(\Pi\left\{X_{\alpha}: \alpha<\boldsymbol{m}\right\}\right)$. We first note that the set $\left\{\beta:\left|X_{\beta}\right|>\boldsymbol{n}\right\}$ must be finite. If it were infinite then $\boldsymbol{n}=$ $\Delta\left(\Pi\left\{X_{\alpha}: \alpha<\boldsymbol{m}\right\}\right) \geqq \boldsymbol{k}>\boldsymbol{n}$, where $\boldsymbol{k}$ is the next cardinal bigger than $\boldsymbol{n}$. Now we clearly have one of the following three cases.

Case I. The set $C=\left\{\beta:\left|X_{\beta}\right|=\boldsymbol{n}\right\}$ is infinite. In this case, the hypothesis of Theorem 1 is obviously satisfied for $X=\Pi\left\{X_{\alpha}: \alpha \in C\right\}$. Hence, $X$ is maximally resolvable. Clearly, $\Delta(X) \geqq \boldsymbol{n}^{\aleph_{0}} \geqq \boldsymbol{n}=\Delta(Y)$, where $Y=\prod\left\{X_{\alpha}: \alpha \notin C\right\}$. Hence, by Lemma $2, X \times Y=\Pi\left\{X_{\alpha}: \alpha<\boldsymbol{m}\right\}$ is maximally resolvable.

Case II. For some $\xi, \Delta\left(X_{\xi}\right) \geqq \boldsymbol{n}$. In this case we must have $\Delta\left(X_{\xi}\right)=\boldsymbol{n}$. Let $\left.\boldsymbol{n}_{1}=\Delta\left(\Pi X_{\alpha}: \alpha \neq \xi\right\}\right)$. Then, it is easily verified that $\boldsymbol{n}=\Delta\left(X_{\xi}\right) \cdot \boldsymbol{n}_{1}=\boldsymbol{n} \cdot \boldsymbol{n}_{1}$. Hence $\boldsymbol{n}_{1} \leqq \boldsymbol{n}$ so that the maximally resolvable 
space $X_{\xi}$ dominates $\Pi\left\{X_{\alpha}: \alpha \neq \xi\right\}$ and $\Pi\left\{X_{\alpha} ; \alpha<\boldsymbol{m}\right\}$ is maximally resolvable by Lemma 2 .

Case III. The set $C=\left\{\beta:\left|X_{\beta}\right| \geqq \boldsymbol{n}\right\}$ is finite and $\Delta\left(X_{\alpha}\right)<\boldsymbol{n}$ for all $\alpha$. The case where $C=\varnothing$ will be seen to be a trivial modification of the case where $C \neq \varnothing$, so assume $C \neq \varnothing$. Put $X=\Pi\left\{X_{\alpha}: \alpha \notin C\right\}$ and $Y=\Pi\left\{X_{\alpha}: \alpha \in C\right\}$. By Lemma $1 Y$ is maximally resolvable. Moreover, it is easily seen that $\left.\boldsymbol{n}=\mid \Pi \Delta\left\{X_{\alpha}\right): \alpha \in C\right\} \mid \cdot \Delta(X)$. But since each $\Delta\left(X_{\alpha}\right)<\boldsymbol{n}$ and $C$ is finite, we must therefore have $\Delta(X)=\boldsymbol{n}$. Now the hypothesis of Theorem 1 can easily be seen to be satisfied for $X$. Firstly, $\left|X_{\beta}\right| \leqq \boldsymbol{n}=\Delta(X)$ for each $\beta$. Secondly, for any finite set $A$ there exists a finite superset $B$ for which

$$
\begin{aligned}
\Delta(X) & =\left|\Pi\left\{\Delta\left(X_{\alpha}\right): \alpha \in B\right\}\right| \cdot\left|\Pi\left\{X_{\alpha}: \alpha \notin B\right\}\right| \\
& =\left|\Pi\left\{\Delta\left(X_{\alpha}\right): \alpha \in A\right\}\right| \cdot\left|\Pi\left\{X_{\alpha}: \alpha \in B-A\right\}\right| \cdot\left|\Pi\left\{X_{\alpha}: \alpha \in B\right\}\right| \\
& =\left|\Pi\left\{\Delta\left(X_{\alpha}\right): \alpha \in A\right\}\right| \cdot\left|\Pi\left\{X_{\alpha}: \alpha \notin A\right\}\right| \\
& =\left|\Pi\left\{X_{\alpha}: \alpha \notin A\right\}\right| .
\end{aligned}
$$

Hence, in Case III, $\Pi\left\{X_{\alpha}: \alpha<\boldsymbol{m}\right\}$ is maximally resolvable, completing the proof.

Theorem 1 gives a particular sufficient condition for an infinite product of arbitrary spaces to be maximally resolvable. The next result gives another, and simpler, sufficient condition.

THEOREM 3. Suppose $\boldsymbol{m} \geqq \boldsymbol{\aleph}_{0}$ and $\boldsymbol{n}=\sup \left\{\left|X_{\alpha}\right|: \alpha<\boldsymbol{m}\right\} . \quad$ If $\left\{\alpha:\left|X_{\alpha}\right|=\boldsymbol{n}\right\}$ is empty or infinite, then II $\left\{X_{\alpha}: \alpha<\boldsymbol{m}\right\}$ is maximally resolvable.

Proof. The proof consists of verifying the hypotheses of Theorem 1. If $A$ is any finite subset of $\boldsymbol{m}$, then there exists $\beta<\boldsymbol{m}$ such that $\max \left\{\left|X_{\alpha}\right|: \alpha \in A\right\} \leqq\left|X_{\beta}\right|$. Hence $\left|X_{\beta}\right| \geqq\left|\Pi\left\{X_{\alpha}: \alpha \in A\right\}\right|$ and consequently

$$
\begin{aligned}
\left|\Pi\left\{X_{\alpha}: \alpha \notin A\right\}\right| & \geqq\left|\Pi\left\{X_{\alpha}: \alpha<\boldsymbol{m}\right\}\right| \\
& \geqq \Delta\left(\Pi\left\{X_{\alpha}: \alpha<\boldsymbol{m}\right\}\right) .
\end{aligned}
$$

Secondly, for some finite set $B, \Delta\left(\Pi\left\{X_{\alpha}: \alpha<\boldsymbol{m}\right\}\right) \geqq\left|\Pi\left\{X_{\alpha}: \alpha \notin B\right\}\right| \geqq \boldsymbol{n}$, whether $\left\{\alpha:\left|X_{\alpha}\right|=\boldsymbol{n}\right\}$ be infinite or empty. Hence, II $\left\{X_{\alpha}: \alpha<\boldsymbol{m}\right\}$ is maximally resolvable.

The previous theorems naturally suggest the question: is the product of two spaces, one of which is maximally resolvable, again maximally resolvable? Unfortunatelly we are unable to settle this question, a discussion of which appears in $\S 4$. However, we are able 
to obtain the following partial results.

Theorem 4. Suppose $\Delta(X)=\boldsymbol{\aleph}_{\alpha}$ and $|Y|=\Delta(Y)=\boldsymbol{\aleph}_{\alpha+1}$. Then $X \times Y$ is maximally resolvable whenever $X$ is maximally resolvable.

Proof. Well-order $Y$ as $\left\{\boldsymbol{r}_{\beta}: \beta<\boldsymbol{\aleph}_{\alpha+1}\right\}$, and for each $\beta<\boldsymbol{\aleph}_{\alpha+1}$ put $B_{\beta}=Y-\left\{r_{\gamma}: \gamma<\beta\right\}$. Then each point in $Y$ belongs to at most $\boldsymbol{\aleph}_{\alpha}$ members of $\left\{B_{\beta}: \beta<\boldsymbol{\aleph}_{\alpha+1}\right\}$. Hence, for any $y \in Y$ we can enumerate those members of $\left\{B_{\beta}: \beta<\boldsymbol{\aleph}_{\alpha+1}\right\}$ which contain $y$ as $\left\{B_{y(\xi)}: \xi<\boldsymbol{m}\right\}$ for some cardinal $\boldsymbol{m} \leqq \boldsymbol{\aleph}_{\alpha}$. Now let $\left\{X_{\mu}: \mu<\boldsymbol{\aleph}_{\alpha}\right\}$ be a resolution for $X$. For each $\beta<\boldsymbol{\aleph}_{\alpha+1}$ we put $A_{\beta}=\cup\left\{\{y\} \times X_{\mu}\right.$ : there exists $\mu$ such that $B_{y(\mu)}=B_{\beta}$. It is easily checked that $\left\{A_{\beta}: \beta<\boldsymbol{\aleph}_{\alpha+1}\right\}$ gives a maximal resolution for $X \times Y$, completing the proof.

As an immediate consequence of Theorem 4 we obtain by induction

Corollary 1. If $\Delta(X)=\boldsymbol{\aleph}_{\alpha}$ and $\left|Y_{i}\right|=\Delta\left(Y_{i}\right)=\boldsymbol{\aleph}_{\alpha+i}$ for $i=$ $1, \cdots, n$, then $X \times Y_{1} \times \cdots \times Y_{n}$ is maximally resolvable whenever $X$ is maximally resolvable.

Next we present two theorems, the first of which generalizes Theorem 4 and Corollary 1 to infinite products.

Theorem 5. Suppose $\left|X_{\alpha}\right|=\Delta\left(X_{\alpha}\right)=\boldsymbol{\aleph}_{\alpha}$ whenever $\mu \leqq \alpha<\xi$. It $X_{\nu}$ is maximally resolvable for some $\nu, \mu \leqq \nu<\xi$, then

$$
\Pi\left\{X_{\alpha}: \mu \leqq \alpha<\xi\right\}
$$

is maximally resolvable.

Proof. We divide the proof into two parts according to whether $\nu=\mu$ or $\nu>\mu$.

Part I. $\nu=\mu$. Without loss of generality we may assume that $\mu=0$. If $\xi$ has no last member (i.e., $\xi$ is a limit ordinal), then Theorem 1 gives maximal-resolvability. Under the assumption that $\xi$ has a last member then, it suffices to prove by induction on $\xi$ that for each $\beta<\xi, \Pi\left\{X_{\alpha}: \alpha \leqq \beta\right\}$ is maximally resolvable. By hypothesis this assertion is true for $\beta=0$. Suppose it is true for each $\gamma<\beta$. Then we have two cases.

Case I. $\beta$ is a nonlimit ordinal. Then in particular

$$
\Pi\left\{X_{\alpha}: \alpha<\beta\right\}=\Pi\left\{X_{\alpha}: \alpha \leqq \beta-1\right\},
$$


which is maximally resolvable by the inductive hypothesis. Also, $\left|\Pi\left\{X_{\alpha}: \alpha<\beta\right\}\right| \geqq\left|X_{\beta-1}\right|=\boldsymbol{\aleph}_{\beta-1}$. In case $\left|\Pi\left\{X_{\alpha}: \alpha<\beta\right\}\right|=\aleph_{\beta-1}$ we apply Theorem 4. On the other hand, if $\left|\mathrm{II}\left\{X_{\alpha}: \alpha<\beta\right\}\right|>\boldsymbol{\aleph}_{\beta-1}$, then

$$
\Delta\left(\Pi\left\{X_{\alpha}: \alpha<\beta\right\}\right)=\left|\Pi\left\{X_{\alpha}: \alpha<\beta\right\}\right| \geqq \boldsymbol{\aleph}_{\beta}=\Delta\left(X_{\beta}\right)
$$

so that $\Pi\left\{X_{\alpha}: \alpha<\beta\right\}$ dominates $X_{\beta}$. So in either case $\Pi\left\{X_{\alpha}: \alpha \leqq \beta\right\}$ is maximally resolvable.

Case II. $\beta$ is a limit ordinal. Then we have $\left|\Pi\left\{X_{a}: \alpha<\beta\right\}\right|=$ $\boldsymbol{\aleph}_{\beta}^{i \beta \mid} \geqq \boldsymbol{\aleph}_{\beta}=\Delta\left(X_{\beta}\right)$. By Theorem 3, $\Pi\left\{X_{a}: \alpha<\beta\right\}$ is maximally resolvable. Hence, $\Pi\left\{X_{\alpha}: \alpha<\beta\right\}$ dominates $X_{\beta}$ so that $\Pi\left\{X_{\alpha}: \alpha \leqq \beta\right\}$ is maximally resolvable.

Part II. $\nu>\mu$. Then $\nu=\lambda+n$ where $\lambda$ is a limit ordinal and $n$ is a natural number. Then we have two cases.

Case I. $\mu<\lambda$. Then $\Pi\left\{X_{\alpha}: \mu \leqq \alpha<\lambda\right\}$ is maximally resolvable by Theorem $3, \Pi\left\{X_{\alpha}: \nu \leqq \alpha<\xi\right\}$ is maximally resolvable by Part I, and finally the finite product $\Pi\left\{X_{\alpha}: \lambda \leqq \alpha<\nu\right\}$ is maximally resolvable because it is dominated by $X_{\nu}$. Hence, by Lemma 1 , $\Pi\left\{X_{\alpha}: \mu \leqq \alpha<\xi\right\}$ is maximally resolvable.

Case II. $\lambda \leqq \mu$. Then $\Pi\left\{X_{\alpha}: \nu \leqq \alpha<\xi\right\}$ is maximally resolvable by Part I and the finite product $\Pi\left\{X_{a}: \mu \leqq \alpha<\nu\right\}$ is maximally resolvable because it is dominated by $X_{\nu}$. Hence, by Lemma 1 again, $\Pi\left\{X_{\alpha}: u \leqq \alpha<\xi\right\}$ is maximally resolvable, completing the proof.

Theorem 6. Suppose $\left|X_{\alpha}\right| \leqq\left|X_{\beta}\right|=\Delta\left(X_{\beta}\right)$ whenever $\alpha \leqq \beta \leqq \xi$. If $X_{\xi}$ is maximally resolvable, then $\Pi\left\{X_{\alpha}: \alpha \leqq \xi\right\}$ is maximally resolvable.

Proof. Define an equivalence relation $\approx$ on $\xi+1$ by $\alpha \approx \beta$ if and only if $\left|X_{\alpha}\right|=\left|X_{\beta}\right|$. Then let $\Gamma$ be the set of all first members of the $\approx$ equivalence classes. Let $B$ be the set of all $\gamma \in \Gamma$ such that the interval $[\gamma, \xi]$ intersects only finitely many equivalence classes. Let $\lambda$ be the first member of $B$ and let $\left\{A_{1}{ }^{\prime} \cdots, A_{n}\right\}$ be the collection of those equivalence classes which intersect $[\lambda, \xi]$ and assume $\xi \in A_{n}$. If any $A_{j}$ is infinite, then $\Pi\left\{X_{\alpha}: \alpha \in A_{j}\right\}$ is maximally resolvable by Theorem 3. However, $\Pi\left\{X_{\alpha}: \alpha \in A_{n}\right\}$ is maximally resolvable even if $A_{n}$ is finite, because in that case $\left.\Delta\left(\Pi X_{\alpha}: \alpha \in A_{n}-\{\xi\}\right\}\right)=\Delta\left(X_{\xi}\right)$ so that $X_{\xi}$ dominates $\Pi\left\{X_{\alpha}: \alpha \in A_{n}-\{\xi\}\right\}$. Now if any $A_{i}$ is finite we 
obviously have $\Delta\left(\Pi\left\{X_{\alpha}: \alpha \in A_{i}\right\}\right) \leqq \Delta\left(\left\{\Pi_{\alpha}: \alpha \in A_{n}\right\}\right)$ so that

$$
\Pi\left\{X_{\alpha}: \alpha \in A_{n}\right\}
$$

dominates $\Pi\left\{X_{\alpha}: \alpha \in A_{i}\right\}$. It follows then that $\Pi\left\{X_{\alpha}: \lambda \leqq \alpha \leqq \xi\right\}$ is maximally resolvable by Lemma 1 . Obviously, either $\lambda=0$ or $\lambda$ is infinite. In the latter case, $\Pi\left\{X_{\alpha}: \alpha<\lambda\right\}$ can easily seen to be maximally resolvable by Theorem 3 . Now, applying Lemma 1 we conclude that $\Pi\left\{X_{\alpha}: \alpha \leqq \xi\right\}$ is maximally resolvable.

3. Other topologies on the product. In this section we consider a product space in both the box $(\mathscr{B})$ and the generalized product $(\mathscr{G})$ topologies and show that the product of maximally resolvable spaces is again maximally resolvable with respect to both $\mathscr{B}$ and $\mathscr{C}$.

This result for the box topology is an immediate generalization of Lemma 1.

THEOREM 7. If each $X_{a}$ is maximally resolvable, then

$$
\left(\Pi\left\{X_{a}: \alpha<\boldsymbol{m}\right\}, \mathscr{B}\right)
$$

is maximally resolvable.

Proof. Obviously $J\left(\Pi\left\{X_{\alpha}: \alpha<\boldsymbol{m}\right\}\right)=\left|\Pi\left\{d\left(X_{\alpha}\right): \alpha<\boldsymbol{m}\right\}\right|$. For each $\alpha<\boldsymbol{m}$ let $\left\{A_{\beta}^{\alpha}: \beta<\Delta\left(X_{\alpha}\right)\right\}$ denote a maximal resolution for $X_{\alpha}$. For any $f \in \Pi\left\{\Delta\left(X_{\alpha}\right): \alpha<\boldsymbol{m}\right\}$ put $A_{f}=\Pi\left\{A_{f(\alpha)}^{\alpha}: \alpha<\boldsymbol{m}\right\}$. Then it is easily checked that $\left\{A_{f}: f \in \Pi\left\{\Delta\left(X_{\alpha}\right): \alpha<\boldsymbol{m}\right\}\right\}$ gives a maximal resolution for $\Pi\left\{X_{\alpha}: \alpha<\boldsymbol{m}\right\}$ with respect to $\mathscr{B}$.

The next result is the generalization of Theorem 1 to the generalized product topology.

Theorem 8. Suppose $\boldsymbol{m} \geqq \boldsymbol{\aleph}_{0}$ and $\left|X_{\alpha}\right| \geqq 2$ for each $\alpha<\boldsymbol{m}$. If for each small subset $A$ of $\boldsymbol{m}$

$$
\begin{aligned}
\left|\Pi\left\{X_{\alpha}: \alpha \in A\right\}\right| & \leqq \Delta\left(\Pi\left\{X_{\alpha}: \alpha<\boldsymbol{m}\right\}, \mathscr{C}\right) \\
& \leqq\left|\Pi\left\{X_{\alpha}: \alpha \notin A\right\}\right|,
\end{aligned}
$$

then ( $\left.\left.I X_{\alpha}: \alpha<\boldsymbol{m}\right\}, \mathscr{G}\right)$ is maximally resolvable.

Proof. Denote by $\mathscr{S}$ the set of all small subsets of $\boldsymbol{m}$ and put $\left.\left.\mathscr{A}=\cup\left\{\Pi X_{\alpha}: \alpha \in B\right\}\right\}: B \in \mathscr{S}\right\}$. Letting $\boldsymbol{n}=\mathcal{A}\left(\Pi\left\{X_{\alpha}: \alpha<\boldsymbol{m}\right\}, \mathscr{G}\right)$ we have $|\mathscr{A}| \leqq|\mathscr{S}| \cdot \sup \left\{\left|\Pi\left\{X_{\alpha}: \alpha \in B\right\}\right|: B \in \mathscr{S}\right\} \leqq 2^{m} \cdot \boldsymbol{n} \leqq \boldsymbol{n} \cdot \boldsymbol{n}=\boldsymbol{n}$. Defining $C_{A}$ for $A \in \mathscr{A}$ as in Theorem 1 we have

$$
\left|C_{A}\right|=\left|\amalg\left\{X_{\alpha}: \alpha \notin \beta\right\}\right| \geqq \boldsymbol{n}
$$


for some $B \in \mathscr{S}$. The rest of the proof is the same as in Theorem 1 .

Lemma 3. Suppose $\boldsymbol{m}$ is infinite and $\boldsymbol{n}=\Delta\left(\Pi\left\{X_{\alpha}: \alpha<\boldsymbol{m}\right\}, \mathscr{G}\right)$. If $\boldsymbol{n} \leqq\left|\Pi\left\{\Delta\left(X_{\alpha}\right): \alpha<\boldsymbol{m}\right\}\right|$ or $\left|\Pi\left\{X_{\alpha}: \alpha \in A\right\}\right| \leqq \boldsymbol{n}$ for each small subset $A$ of $\boldsymbol{m}$, then $\left(\Pi\left\{X_{a}: \alpha<\boldsymbol{m}\right\}, \mathscr{G}\right)$ is maximally resolvable whenever each $X_{a}$ is maximally resolvable.

Proof.

Case I. Suppose $\boldsymbol{n} \leqq\left|\Pi\left\{\Delta\left(X_{\alpha}\right): \alpha<\boldsymbol{m}\right\}\right|$. In this case the proof of Theorem 7 shows that $\Pi\left\{X_{\alpha}: \alpha<\boldsymbol{m}\right\}$ is maximally resolvable with respect to $\mathscr{G}$.

Case II. Suppose $\boldsymbol{n} \geqq\left|\Pi\left\{X_{\alpha}: \alpha \in A\right\}\right|$ for each small subset $A$ of $\boldsymbol{m}$. Now, if for each small subset $A$ we had $\boldsymbol{n} \leqq\left|\Pi\left\{X_{\alpha}: \alpha \notin A\right\}\right|$, we could apply Theorem 8 . So let us suppose that for some small subset $A$ of $\boldsymbol{m},\left|\Pi\left\{X_{\alpha}: \alpha \notin A\right\}\right|<\boldsymbol{n}$. However, for some small superset $B$ of $A$ we have $\boldsymbol{n}=\left|\Pi\left\{\Delta\left(X_{\alpha}\right): \alpha \in B\right\}\right| \cdot\left|\Pi\left\{X_{\alpha}: \alpha \in \boldsymbol{m}-B\right\}\right|$, from which we conclude that $\boldsymbol{n}=\left|\Pi\left\{\Delta\left(X_{\alpha}\right): \alpha \in B\right\}\right|$. But the induced $\mathscr{G}$-topology on $\Pi\left\{X_{\alpha}: \alpha \in B\right\}$ coincides with the $\mathscr{B}$-topology in which, by Theorem 7, $\Pi\left\{X_{\alpha}: \alpha \in B\right\}$ is maximally resolvable. Let $\left\{A_{\alpha}\right\}_{\alpha<n}$ be the resolvants for $\Pi\left\{X_{\alpha}: \alpha \in B\right\}$. Then $\left\{A_{\alpha} \times \Pi\left\{X_{\beta}: \beta \neq \alpha\right\}\right\}_{\alpha<n}$ gives a maximal resolution for $\left(\Pi\left\{X_{\alpha}: \alpha<\boldsymbol{m}\right\}, \mathscr{G}\right)$.

Next we need the following purely set-theoretic lemma which is a generalization of the cardinal arithmetic property that $\prod_{\alpha<m} \boldsymbol{\aleph}_{\alpha}=\boldsymbol{\aleph}_{m}^{|m|}$.

Lemma 4. Suppose $\boldsymbol{k}$ is a function from an infinite cardinal $\boldsymbol{m}$ into some set of cardinals. If $\sup \left\{\boldsymbol{k}_{\alpha}: \alpha \in \boldsymbol{m}-A\right\} \geqq \boldsymbol{n}$ for each small subset $A$ of $\boldsymbol{m}$, then $\left|\mathrm{II}\left\{\boldsymbol{k}_{\alpha}: \alpha \in m\right\}\right| \geqq \boldsymbol{n}^{\boldsymbol{m}}$.

Proof. Let $B=\left\{\alpha: \boldsymbol{k}_{\alpha} \geqq \boldsymbol{n}\right\}$. If $|B| \geqq \boldsymbol{m}$, then obviously

$$
\left|\Pi\left\{\boldsymbol{k}_{\alpha}: \alpha \in \boldsymbol{m}\right\}\right| \geqq \boldsymbol{n}^{\boldsymbol{m}} \text {. }
$$

So let us assume that $|B|<\boldsymbol{m}$. Since $|\boldsymbol{m}-B|=\boldsymbol{m}$ and finite unions of small sets are small, without loss of generality we can assume that $B=\varnothing$.

Since the range of $\boldsymbol{k}$ is a subset of the ordinal $\boldsymbol{n}$ it is well-ordered by " $\varepsilon$ " and therefore is order isomorphic to some ordinal $\gamma$, which is necessarily a limit ordinal. Let $e$ be the isomorphism from $\gamma$ onto the range of $\boldsymbol{k}$ and define, for each $\alpha \in \gamma, E_{\alpha}=\left\{\beta: \boldsymbol{k}_{\beta}=e(\alpha)\right\}$. Now let $\mathscr{A}$ consist of all subsets $A$ of $\gamma+1$ which satisfy the following three properties : (1) $0 \in A$; (2) $\alpha+\boldsymbol{m} \in A$ whenever $\alpha \in A$ and $\alpha+\boldsymbol{m} \in \gamma+1$; (3) $B \subseteq A$ implies $\cup B=\sup B \in A$. Since $\gamma+1 \in \mathscr{A}$, $\mathscr{A}$ is nonvoid, 
and we can put $\Gamma=\cap \mathscr{A}$. Obviously $\Gamma$ satisfies properties (1) through (3). Let $C=\Gamma-\{\gamma\}$. Then $\xi=\cup C=\sup C \in \Gamma$ and $\gamma=\xi+\mu$ where $\mu \leqq \boldsymbol{m}$.

Case I. Suppose $\mu=\boldsymbol{m}$. Then the set $D=\{\xi+\alpha: \alpha<\boldsymbol{m}\}$ is order isomorphic to $\boldsymbol{m}$. Since $\boldsymbol{m} \cdot \boldsymbol{m}=\boldsymbol{m}$ it follows that $\boldsymbol{m}$ contains $\boldsymbol{m}$ pairwise disjoint cofinal subsets. Consequently $D$ has a family $\left\{D_{\alpha}\right\}_{\alpha<m}$ of pairwise disjoint subsets, each of which is cofinal in $D$ and $\gamma$. Hence, each $\boldsymbol{k}^{-1} e\left(D_{\alpha}\right)=F_{\alpha}$ is cofinal in $\boldsymbol{n}$ so that $\left|\prod\left\{\boldsymbol{k}_{\beta}: \beta \in F_{\alpha}\right\}\right| \geqq \boldsymbol{n}$. Therefore,

$$
\left|\Pi\left\{\boldsymbol{k}_{\beta}: \beta<m\right\}\right| \geqq\left|\Pi_{\alpha<\boldsymbol{m}}\left(\Pi\left\{\boldsymbol{k}_{\beta}: \beta \in F_{\alpha}\right\}\right)\right| \geqq \boldsymbol{n}^{\boldsymbol{m}} .
$$

Case II. Suppose $\mu=0$. For each $\alpha<\boldsymbol{m}$, let $D_{\alpha}=\{\sigma+\alpha: \sigma \in \Gamma\}$. Then $\left\{D_{\alpha}\right\}_{\alpha<m}$ is a family of pairwise disjoint subsets of $\Gamma$, each of which is cofinal in $\gamma$. The remainder of the proof is as in Case I.

Case III. Suppose $0<\mu<\boldsymbol{m}$. Then for each $\nu<\mu$,

$$
\sup \left\{\left|E_{\beta}\right|: \xi+\nu \leqq \beta \leqq \gamma\right\}=\boldsymbol{m} .
$$

If this were not the case then, for some $\boldsymbol{t}<\boldsymbol{m}$ and some $\nu<\mu$, $\sup \left\{\left|E_{\beta}\right|: \xi+\nu \leqq \beta<\gamma\right\} \leqq \boldsymbol{t}<\boldsymbol{m}$. Hence the set

$$
A=U\left\{E_{\beta}: \xi+\nu \leqq \beta<\gamma\right\}
$$

is such that $|A| \leqq t \cdot|\mu|<\boldsymbol{m}$, i. e. $A$ is small. Then

$$
\sup \left\{\boldsymbol{k}_{\alpha}: \alpha \in \boldsymbol{m}-A\right\} \leqq e(\xi+\nu)<\boldsymbol{n},
$$

which is a contradiction. Next, enumerate each $E_{\beta}$ as $\left\{e_{\beta}^{\alpha}: \alpha<\left|E_{\beta}\right|\right\}$, and for each $\alpha<\boldsymbol{m}$ define

$$
A_{\alpha}=\left\{x: \text { there exists } \beta \geqq \xi \text { such that } x=e_{\beta}^{\alpha}\right\} .
$$

It is readily verified that $\left\{A_{\alpha}\right\}_{\alpha<m}$ is a family of nonvoid pairwise disjoint subset of $\boldsymbol{m}$ such that each $k\left(A_{\alpha}\right)$ is cofinal in $\boldsymbol{n}$. Hence, $\left|\Pi\left\{\boldsymbol{k}_{\hat{\beta}}: \beta \in A_{\alpha}\right\}\right| \geqq \boldsymbol{n}$ for each $\alpha<\boldsymbol{m}$, and

$$
\left|\Pi\left\{\boldsymbol{k}_{\beta}: \beta<\boldsymbol{m}\right\}\right| \geqq\left|\prod_{\alpha<\boldsymbol{m}}\left(\Pi\left\{\boldsymbol{k}_{\beta}: \beta \in A_{\alpha}\right\}\right)\right| \geqq \boldsymbol{n}^{\boldsymbol{m}},
$$

which completes the proof.

THEOREM 9. If each $X_{\alpha}$ is maximally resolvable, then

$$
\left(\Pi\left\{X_{\alpha}: \alpha<m\right\}, \mathscr{G}\right)
$$

is maximally resolvable. 
Proof. Let us assume that $\left(\Pi\left\{X_{\alpha}: \alpha<\boldsymbol{m}\right\}, \mathscr{G}\right)$ is not maximally resolvable. Then, for each small subset $B$ of $\boldsymbol{m}$ the following inequality is satisfied for some small subset $D$ of $\boldsymbol{m}-B$ :

$$
\left|\Pi\left\{\Delta\left(X_{\alpha}\right): \alpha \in \boldsymbol{m}-B\right\}\right|<\Delta\left(\Pi\left\{X_{\alpha}: \alpha \in \boldsymbol{m}-B\right\}\right)<\left|\Pi\left\{X_{\alpha}: \alpha \in D\right\}\right| .
$$

If this were not the case then $\Pi\left\{X_{\alpha}: \alpha \in \boldsymbol{m}-B\right\}$ would be maximally resolvable by Lemma 3. However, $\Pi\left\{X_{\alpha}: \alpha \in B\right\}$ is also maximally resolvable by Theorem 7 . Hence, $\Pi\left\{X_{\alpha}: \alpha<\boldsymbol{m}\right\}$ would be maximally resolvable with respect to $\mathscr{G}$, a contradiction.

By taking $B=\varnothing$ in the above assumption we have for some small subset $A_{0}$ of $\boldsymbol{m}$ :

$$
\left|\Pi\left\{\Delta\left(X_{\alpha}\right): \alpha<\boldsymbol{m}\right\}\right|<\Delta\left(\Pi\left\{X_{\alpha}: \alpha<\boldsymbol{m}\right\}\right)<\left|\Pi\left\{X_{\alpha}: \alpha \in A_{0}\right\}\right| .
$$

Let $C$ be a small subset of $\boldsymbol{m}$ for which

$$
\Delta\left(\Pi\left\{X_{\alpha}: \alpha<\boldsymbol{m}\right\}\right)=\left|\Pi\left\{\Delta\left(X_{\alpha}\right): \alpha \in C\right\}\right| \cdot\left|\Pi\left\{X_{\alpha}: \alpha \notin C\right\}\right| .
$$

Then it follows that we can find a large subset $M_{1}$ of $\boldsymbol{m}$ with $\sup \left\{\left|X_{\alpha}\right|: \alpha \in M_{1}\right\}<\boldsymbol{n}_{0}$ where $\boldsymbol{n}_{0}=\sup \left\{\left|X_{\alpha}\right|: \alpha \in A_{0}\right\}$. If this were not the case then we would have $\sup \left\{\left|X_{\alpha}\right|: \alpha \in \boldsymbol{m}-C-A\right\} \geqq \boldsymbol{n}_{0}$ for every small subset $A$ of $\boldsymbol{m}$. Then an application of Lemma 4 yields

$$
\Delta\left(\Pi\left\{X_{\alpha}: \alpha<\boldsymbol{m}\right\}\right) \geqq \Pi\left\{\left|X_{\alpha}\right|: \alpha \in \boldsymbol{m}-C\right\} \geqq \boldsymbol{n}_{0}^{\boldsymbol{m}} .
$$

But this contradicts the original assumption that

$$
\Delta\left(\Pi\left\{X_{\alpha}: \alpha<\boldsymbol{m}\right\}\right)<\left|\Pi\left\{X_{\alpha}: \alpha \in A_{0}\right\}\right| \leqq \boldsymbol{n}_{0}^{\left|A_{0}\right|} \leqq \boldsymbol{n}_{0}^{n} .
$$

Hence, a large subset $M_{1}$ of $\boldsymbol{m}$ exists such that $\sup \left\{\left|X_{\alpha}\right|: \alpha \in M_{1}\right\}<\boldsymbol{n}_{0}$.

Now applying the original assumption to the set $B=\boldsymbol{m}-M_{1}$ we obtain a small subset $A_{1}$ of $M_{1}$ for which

$$
\left|\Pi\left\{\Delta\left(X_{\alpha}\right): \alpha \in M_{1}\right\}\right|<\Delta\left(\Pi\left\{X_{\alpha}: \alpha \in M_{1}\right\}\right)<\left|\Pi\left\{X_{\alpha}: \alpha \in A_{1}\right\}\right| .
$$

Now put $\boldsymbol{n}_{1}=\sup \left\{\left|X_{\alpha}\right|: \alpha \in A_{1}\right\}$. Then $\boldsymbol{n}_{1} \leqq \sup \left\{\left|X_{\alpha}\right|: \alpha \in M_{1}\right\}<\boldsymbol{n}_{0}$. Repeating the argument above we then find a large subset $M_{2}$ of $M_{1}$ for which $\sup \left\{\left|X_{\alpha}\right|: \alpha \in M_{2}\right\}<\boldsymbol{n}_{1}$.

Continuing in this way by induction we obtain sequences $\left\{M_{k}\right\}_{k=0}^{\infty}$, $\left\{A_{k}\right\}_{k=0}^{\infty}$ and $\left\{\boldsymbol{n}_{k}\right\}_{k=0}^{\infty}$ such that $M_{k+1}$ is a large subset of $M_{k}$ (where $\left.M_{0}=\boldsymbol{m}\right), A_{k}$ is a small subset of $M_{k}, \quad \boldsymbol{n}_{k}=\sup \left\{\left|X_{\alpha}\right|: \alpha \in M_{k}\right\}$ and $\boldsymbol{n}_{k+1}<\boldsymbol{n}_{k}$ for each $k$. But this gives a contradiction since there is no (strictly) decreasing infinite sequence of cardinals, which completes the proof.

An interesting question is whether or not maximal-resolvability is 
preserved under other natural topologies defined on the product. For example, let $\mathscr{E}$ be any family of subsets of the given index set $\boldsymbol{m}$ which contains $\varnothing$ and is closed under finite unions. Then the family $\mathscr{U}$ of all subsets $\Pi\left\{U_{\alpha} \mid \alpha \in \boldsymbol{m}\right\}$ of $\Pi\left\{X_{\alpha} \mid \alpha \in \boldsymbol{m}\right\}$ for which $U_{\alpha}=X_{\alpha}$ except for $\alpha$ belonging to a set in $\mathscr{E}$ is a base for some topology $\tau(\mathscr{E})$. If $\mathscr{E}$ is taken to be the family of finite (resp. small, all) subsets of the index set, then $\tau(\mathscr{E})$ is $\mathscr{P}$ (resp. $\mathscr{C}, \mathscr{B})$.

A $\tau(\mathscr{B})$ different from $\mathscr{G}$ and $\mathscr{P}$ can be obtained by taking $\boldsymbol{m}$ to be a limit cardinal and $\mathscr{E}$ to be the set of all subsets of $\boldsymbol{m}$ of cardinality less than or equal to $k$ (or alternatively less than $k$ if $\boldsymbol{k}>\boldsymbol{W}_{0}$ ), where $\boldsymbol{k}$ is an infinite cardinal less than $\boldsymbol{m}$. Neither the proof of Theorem 2 nor that of Theorem 9 appears to generalize to this particular $\tau(\mathscr{E})$. It may be noted that if a product space with respect to one of these $\tau(\mathscr{E})$ topologies should fail to be maximally resolvable, then the three unanswered questions of $\S 5$ would be settled.

4. Minimal Spaces. Before posing and discussing some unsolved problems it is necessary to review the concept of a minimal space as originally introduced and studied by Hewitt [2] and Katětov [3]. A dense-in-itself space $(\mathrm{S}, \sigma)$ is called minimal [3] if for any dense-initself $(T, \tau)$ any one-to-one continuous function from $T$ onto $S$ is necessarily a homeomorphism. Minimal spaces form a subclass of the $\tau$-maximal spaces of Hewitt [2]. For our purposes, the main results about minimal spaces are the following:

Theorem 10. (Katětov [3], Hewitt [2]) Any dense-in-itself Hausdorff space $(X, \tau)$ has a larger topology $\sigma$ such that $(X, \sigma)$ is minimal.

Proof. (Katétov [3]) Let $\mathscr{D}$ be the collection of all subsets of $X$ each of which is dense-in-itself. Let $\mathscr{A}$ consist of all families $\mathscr{C}$ of subsets of $X$ such that $\tau \subseteq \mathscr{C} \subseteq \mathscr{D}$ and $\mathscr{C}$ is closed under finite intersections. Applying Zorn's Lemma to $(\mathscr{A}, \subseteq)$ one obtains a maximal member of $\mathscr{A}$ which is easily seen to yield the desired minimal topology.

Corollary 2. (Katětov [3], Hewitt [2]) Any dense subset of a minimal space is open; hence, there exists no pair of disjoint, dense subsets.

Proof. If $A$ is a dense set which is not open, then the family of all sets of the form $(A \cap G) \cup H$, where $G$ and $\mathrm{H}$ are open, gives a larger topology, contradicting the maximality of the minimal topology.

Let us now modify the definition of a minimal space to require 
that both $\Delta(S)$ and $\Delta(T)$ be equal to $\boldsymbol{m}$. Such a space will be called m-minimal. Then analogously to the above results we obtain

THEOREM 11. Any dense-in-itself Hausdorff space has a larger topology which is $\Delta(X)$-minimal.

Proof. The proof is the same as that of Theorem 10 except that we add the requirement that $D \in \mathscr{D}$ implies $|D| \geqq \Delta(X)$.

Corollary 3. In an $\boldsymbol{m}$-minimal space each $\boldsymbol{m}$-dense subset is open; each dense set is $\boldsymbol{m}$-dense and open; there exists no pair of disjoint, dense subsets.

Proof. The proof that each $\boldsymbol{m}$-dense subset is open is similar to the proof of Corollary 2. Suppose $A$ is dense and $U$ is open such that $|U \cap A|<\boldsymbol{m}$. Then $U-A$ would be $\boldsymbol{m}$-dense in $U$ and hence be an open set missing $A$, a contradiction.

As mentioned in $\S 2$ an infinite-fold product of a minimal space cannot be minimal. We can actually strengthen this remark to the following result.

THEOREM 12. Suppose $\boldsymbol{m} \geqq \boldsymbol{\aleph}_{0}$ and $\left|X_{\alpha}\right| \geqq 2$ for each $\alpha<\boldsymbol{m}$. Then $\left(\Pi\left\{X_{\alpha}: \alpha<\boldsymbol{m}\right\}, \mathscr{P}\right)$ contains infinitely many pairwise disjoint dense subsets.

Proof. Suppose for some infinite subset $A$ of $\boldsymbol{m}$,

$$
B=\left\{\alpha \in A:\left|X_{\alpha}\right|=\boldsymbol{n}\right\}
$$

is void or infinite, where $\boldsymbol{n}=\sup \left\{\left|X_{\alpha}\right|: \alpha \in A\right\}$. Then $\Pi\left\{X_{\alpha}: \alpha \in A\right\}$ is maximally resolvable by Theorem 3 and hence, contains a sequence $\left\{D_{n}\right\}_{n=1}^{\infty}$ of pairwise disjoint dense subsets. It follows then that $\left\{D_{n} \times \Pi\left\{X_{\alpha}: \alpha \notin A\right\}\right\}_{n=1}^{\infty}$ is a sequence of pairwise disjoint, dense subsets of $\Pi\left\{X_{\alpha}: \alpha<\boldsymbol{m}\right\}$.

So let us suppose that there exists no infinite set $A$ for which the above property holds. Then by induction we can easily construct sequences $\left\{B_{k}\right\}_{k=1}^{\infty}$ and $\left\{\boldsymbol{n}_{k}\right\}_{k=1}^{\infty}$ such that $\boldsymbol{n}_{1}=\sup \left\{\left|X_{\alpha}\right|: \alpha<\boldsymbol{m}\right\}$ and $B_{1}=\left\{\alpha:\left|X_{\alpha}\right|=n_{1}\right\}$ and $\boldsymbol{n}_{k+1}=\sup \left\{\left|X_{\alpha}\right|: \alpha \notin \bigcup_{i=1}^{k} B_{i}\right\}$ and

$$
B_{k+1}=\left\{\alpha \in \boldsymbol{m}-\bigcup_{i=1}^{k} B_{i}:\left|X_{\alpha}\right|=\boldsymbol{n}_{k+1}\right\},
$$

with the properties that each $\boldsymbol{B}_{k}$ is nonvoid and finite and $\boldsymbol{n}_{k+1}<\boldsymbol{n}_{k}$ for each $k$. But this is a contradiction since there is no decreasing 
sequence of cardinals.

Since minimal spaces must contain more than one point, the following is immediate.

Corollary 4. Any infinite product of minimal spaces is not minimal.

5. Some unsolved Problems. The previously mentioned question $\mathrm{Q}_{1}$ : "Is the product of a maximally resolvable space with any other space again maximally resolvable?" is related to two other interesting unsolved questions, namely:

$\mathrm{Q}_{2}$ : Do there exist infinite cardinals $\boldsymbol{n}, \boldsymbol{m}$ and a nonmaximally resolvable space $X$ which has $\boldsymbol{n}$ disjoint, $\boldsymbol{n}$-dense subsets and for which $\boldsymbol{n}<\boldsymbol{m}=\Delta(X)$ ?

$\mathrm{Q}_{3}$ : Does there exist a maximally resolvable space having an open subspace which is not maximally resolvable?

An affirmative answer to $Q_{3}$ was prematurely claimed in [1] as being obvious. Indeed, this is the case whenever $|X|=\Delta(X)$ or $\chi(X) \leqq \Delta(X)$, but in general the problem remains unsolved. In fact, questions $\mathrm{Q}_{2}$ and $\mathrm{Q}_{3}$ are equivalent as shown by

Proposition 1. Question $Q_{2}$ has an affirmative answer if and only if $\mathrm{Q}_{3}$ has an affirmative answer.

Proof. $(\Rightarrow)$ Suppose $(X, \sigma)$ is a nonmaximally resolvable space which has $\boldsymbol{n}$ disjoint, $\boldsymbol{n}$-dense subsets where $\boldsymbol{n}<\Delta(X)$. Choose a set $Y$ disjoint from $X$ such that $|Y|=n$. Equip $Y$ with the topology $\tau$ consisting of all large subsets of $Y$, together with $\Phi$. Put $Z=X \cup Y$ and give $Z$ the topology whose base is $\sigma \cup \tau$. Now $Y$ is obviously maximally resolvable with $\Delta(Y)=\boldsymbol{n}$ so that $\Delta(Z)=\boldsymbol{n}$ and $Z$ is maximally resolvable. Yet $X$ is an open subspace which is not maximally resolvable.

$(\Leftrightarrow)$ Suppose $U$ is a nonmaximally resolvable, open subspace of a maximally resolvable space $\mathrm{X}$. Then $\mathrm{U}$ has $\Delta(X)$ disjoint, $\Delta(X)$-dense subsets, yet $U$ is not maximally resolvable.

If $\boldsymbol{n}$ in $\mathrm{Q}_{2}$ is not required to be infinite, then the answer to $Q_{2}$ would be affirmative, as shown by

EXAMPle 1. There exists a space $Y$, with $\Delta(Y)$ infinite, which has two, but not more than two, disjoint dense subsets.

Proof. Let $X$ be an $\boldsymbol{m}$-minimal space with $\boldsymbol{m} \geqq \boldsymbol{\aleph}_{1}$. Give $\{1,2\}$ the indiscrete topology, and put $Y=X \times\{1,2\}$. Let $Y$ have the ordinary product topology. For any subset $D$ of $X$, let us put 
$D_{i}=D \times\{i\}$ for $i=1$, 2. We shall use Theorem 20 of Hewitt [2], which states that a space is resolvable if and only if each open subset contains a resolvable subspace. (A space is resalvable if it admits two disjoint dense subsets.)

Now suppose $A, B$ and $C$ are three pairwise disjoint, dense subsets of $Y$. Let $U_{1}$ be an open subset of $X_{1}$ which contains no resolvable subspace. In particular, $A \cap X_{1}$ and $B \cap X_{1}$ are not both dense in $U_{1}$, so there exists an open subset $V_{1}$ in $U_{1}$ which misses, say, $A$. Since $V_{1}$ is not resolvable, either $B \cap X_{1}$ or $C \cap X_{1}$ fails to be dense in $V_{1}$. Hence, there exists an open set $W_{1}$ in $V_{1}$ which misses, say, $B$. It follows that $W_{1}$ is an open set which is nonresolvable and is contained entirely in $C$, and of course $W_{2}$ is also nonresolvable. However, since $W_{1} \subset C$ and $A$ and $B$ are dense in $Y$, both $A \cap X_{2}$ and $B \cap X_{2}$ must be dense in $W_{2}$, a contradiction. Therefore, there cannot be more than two disjoint, dense subsets of $Y$. Obviously $X_{1}$ and $X_{2}$ are disjoint dense subsets of $Y$.

If we now let $Y=X \times\{1,2, \cdots, k\}$ ( $X$ as before) and define topologies on $\{1,2, \cdots, k\}$ and $Y$ in the analogous manner, then it is not difficult to show that this space admits $k$, but not $k+1$, disjoint dense subsets.

From these considerations it is clear that the space $Y=X \times N$, where $N$ represents the positive integers with the indiscrete topology, is an obvious candidate for a space which has $\boldsymbol{\aleph}_{0} \boldsymbol{m}$-dense subsets but is not maximally resolvable. However, if $|X|=\boldsymbol{m}=\boldsymbol{\aleph}_{1}$, Theorem 4 shows that $Y$ is maximally resolvable. On the other hand, the case where $\boldsymbol{m} \geqq \boldsymbol{\aleph}_{2}$ is seemingly much more difficult, and we conjecture that in this case $Y$ is not maximally resolvable. If this conjecture is true, then it of course also answers $Q_{2}$ and $Q_{3}$ affirmatively. In general, then, a negative answer to $Q_{1}$ implies positive answers to both $\mathrm{Q}_{2}$ and $\mathrm{Q}_{3}$.

Question $\mathrm{Q}_{1}$ can be restated in a simpler-appearing form in case $|X|=\Delta(X)=\boldsymbol{\aleph}_{0}$, as shown by:

Proposition 2. Suppose $X$ is maximally resolvable with $|X|=$ $\Delta(X)=\boldsymbol{\aleph}_{0}$, and let $\Delta(Y)=\boldsymbol{m} \geqq \boldsymbol{\aleph}_{0}$. Then $X \times Y$ is maximally resolvable if and only if there exists in $Y$ a point-countable family of $\boldsymbol{m}$ distinct $\boldsymbol{m}$-dense subsets.

Proof. If $X \times Y$ is maximally resolvable, then the projections of the resolvants provide such a family in $Y$. On the other hand, the proof of Theorem 4 shows that $X \times Y$ is maximally resolvable when such a family exists.

We conjecture that for sufficiently large $\boldsymbol{m}, \boldsymbol{m}$-minimal spaces can be found to violate the condition of Proposition 2. 


\section{BIBLIOGRAPHY}

1. J. Ceder, On maximally resolvable spaces, Fund. Math. 55 (1964), 87-93.

2. E. Hewitt, A problem of set-theoretic topology, Duke Math. J. 10 (1943), 309-333.

3. M. Katětov, On topological spaces containing no disjoint dense sets (Russian), Mat. Sb. 21 (1947), 3-12.

Received September 28, 1966.

UNIVERSITY OF CALIFORNIA

Santa Barbara, California and

ACADIA UNIVERSITY

WolfVille, Nova Scotia 



\section{PACIFIC JOURNAL OF MATHEMATICS}

\section{EDITORS}

\section{H. SAMELSON}

Stanford University

Stanford, California

J. P. JANS

University of Washington

Seattle, Washington 98105
J. Dugundji

University of Southern California Los Angeles, California 90007

RICHARD ARENS

University of California

Los Angeles, California 90024

\section{ASSOCIATE EDITORS}
E. F. BECKENBACH
B. H. NeumanN
F. WOLF
K. YosidA

\section{SUPPORTING INSTITUTIONS}

UNIVERSITY OF BRITISH COLUMBIA

CALIFORNIA INSTITUTE OF TECHNOLOGY

UNIVERSITY OF CALIFORNIA

MONTANA STATE UNIVERSITY

UNIVERSITY OF NEVADA

NEW MEXICO STATE UNIVERSITY

OREGON STATE UNIVERSITY

UNIVERSITY OF OREGON

OSAKA UNIVERSITY

UNIVERSITY OF SOUTHERN CALIFORNIA
STANFORD UNIVERSITY

UNIVERSITY OF TOKYO

UNIVERSITY OF UTAH

WASHINGTON STATE UNIVERSITY

UNIVERSITY OF WASHINGTON

AMERICAN MATHEMATICAL SOCIETY CHEVRON RESEARCH CORPORATION TRW SYSTEMS

NAVAL ORDNANCE TEST STATION 


\section{Pacific Journal of Mathematics \\ Vol. 22, No. $1 \quad$ January, 1967}

Charles A. Akemann, Some mapping properties of the group algebras of a

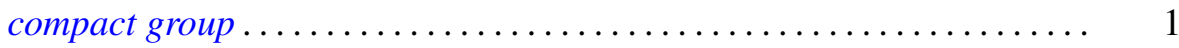

A. V. Boyd, Note on a paper by Uppuluri ..................... 9

Thomas Craig Brown, A semigroup union of disjoint locally finite subsemigroups which is not locally finite .....................

Richard Thomas Bumby and Everett C. Dade, Remark on a problem of

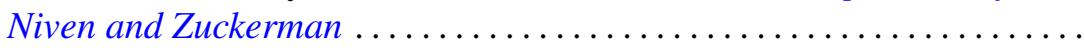

James Calvert, An integral inequality with applications to the Dirichlet problem .........................................

Jack Gary Ceder and Terrance Laverne Pearson, On products of maximally resolvable spaces ....................................

William Guignard Faris, The product formula for semigroups defined by

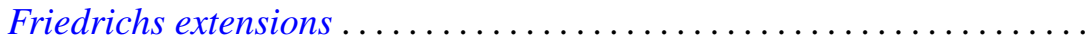

Robert S. Freeman, Closed operators and their adjoints associated with elliptic differential operators ........................ 71

Thomas Lee Hayden, The extension of bilinear functionals ............. 99

Gloria Conyers Hewitt, Limits in certain classes of abstract algebras . . . . . 109

Tilla Weinstein, The dilatation of some standard mappings ........... 117

Mitsuru Nakai, On Evans' kernel ......................... 125

Ernest Levane Roetman, On the biharmonic wave equation ............ 139

Malcolm Jay Sherman, Operators and inner functions ... . .

Walter Laws Smith, On the weak law of large numbers and the generalized elementary renewal theorem

A. J. Ward, On H-equivalence of uniformities: The Isbell-Smith problem 